\title{
Integration of an Adaptive Swing Control into a Neuromuscular Human Walking Model*
}

\author{
Seungmoon Song, Ruta Desai, and Hartmut Geyer
}

\begin{abstract}
Understanding the neuromuscular control underlying human locomotion has the potential to deliver practical controllers for humanoid and prosthetic robots. However, neurocontrollers developed in forward dynamic simulations are seldom applied as practical controllers due to their lack of robustness and adaptability. A key element for robust and adaptive locomotion is swing leg placement. Here we integrate a previously identified robust swing leg controller into a full neuromuscular human walking model and demonstrate that the integrated model has largely improved behaviors including walking on very rough terrain $( \pm 10 \mathrm{~cm})$ and stair climbing $(15 \mathrm{~cm}$ stairs). These initial results highlight the potential of the identified robust swing control. We plan to generalize it to a range of human locomotion behaviors critical in rehabilitation robotics.
\end{abstract}

\section{INTRODUCTION}

Understanding the neuromuscular control underlying human locomotion has the potential to advance the state of the art in different fields. It can lead to new rehabilitation methods [1], [2], provide simulation testbeds which realize virtual experiments difficult or impossible to conduct with human subjects [3]-[5], and deliver practical controllers for humanoid and prosthetic robots [6]-[9]. Since the human neural control architecture is difficult to identify directly, several research groups develop computational models of neuromuscular control to propose and test specific control architectures. For instance, inspired by the observation of central pattern generators (CPGs) in neurophysiological studies [10], [11], neural control architectures based on CPGs and feedback pathways have been proposed in simulation studies and have been found to generate walking and running [12][16]. Similar results have been obtained [17] testing neuromuscular control based on the equilibrium point hypothesis [18] and on interpreting principles of legged dynamics and control with muscle reflexes [19], [20]. However, all these controllers have so far produced only limited robustness and adaptability required for real world applications and, thus, are seldom applied for control in robotic systems.

A key element for robust and adaptive locomotion is swing leg placement; it is, for instance, critical in maintaining gait stability in legged systems that encounter large disturbances. Simplified models of dynamic balance such as the linear inverted pendulum model and the spring-mass model [21][23] can be used to predict these target placements. But these

\footnotetext{
*This work is supported in part by the NSF through the NSF ERC on Quality of Life Technology (EEC 0540865) and by NIH through the NICHD National Center for Medical Rehabilitation Research (1R01HD075492-01)

S. Song, R. Desai, and H. Geyer are with the Robotics Institute, Carnegie Mellon University, 5000 Forbes Avenue, Pittsburgh, PA 15213, USA. \{smsong, rutad, hgeyer\} at cmu.edu
}

models do not reveal how humans control their segmented legs to reach the targets. In a recent study, we have idealized the problem of swing leg placement by studying a double pendulum system that is hinged at the hip and found a control that achieves robust leg placement into arbitrary target points on the ground under large disturbances [24].

Here we integrate this swing leg controller into our full neuromuscular human walking model, and explore its potential of generating different and robust locomotion behaviors. In section II, we briefly review the previous human model and the identified swing leg controller. We then present our work on integrating the two in section III and show in section IV that the resulting model not only generates steady locomotion at normal walking speeds, but also negotiates very rough terrain and climbs stairs. Finally, we discuss future directions of this work in section $\mathrm{V}$.

\section{PREVIOUS NEUROMUSCULAR WALKING MODEL AND ROBUST SWING CONTROL}

\section{A. Previous Neuromuscular Model}

The musculoskeletal system of the previous model consists of 7 segments (trunk, thighs, shanks and feet) and 6 internal degrees of freedom (hip, knee and ankle joints) [19] (Fig. 1). The joints are actuated by seven Hill-type muscle models per leg, five of which are monoarticular muscles (soleus, SOL; tibialis anterior, TA; vastus, VAS; gluteus maximus, GLU; and grouped hip flexors, HFL) and two of which are biarticular ones (gastrocnemius, GAS and hamstring group, HAM). The contractile elements of the muscle models take stimulation signals $S_{m}$ between 0 and 1 , which generate muscle forces that translate into joint torques, $\tau_{m}=F_{m} r_{m}(\varphi)$, where $r_{m}(\varphi)$ estimate the variable moment arms observed in physiology. The ground contacts and joint limits are modeled as nonlinear spring-dampers.

The muscle stimulations $S_{m}$ are the outputs of the neural control architecture of this model. The full control network can be categorized into four control groups based on their functionalities: trunk balance, stance, swing initiation, and swing control. The trunk balance control is active proportionally to the load the leg is bearing during the stance phase; the stance control is active throughout the stance phase; swing initiation is active during the double support phase of late stance phase; and the swing control is active during the swing phase (Fig. 1-a). Most of the sensory reflex pathways are local positive force or length feedbacks, $F+$ or $L+$ (Fig. 1b). These sensory and stimulation signals are time-delayed, to model neural transport delays. (See [19] for more details on this model.) 
(a)

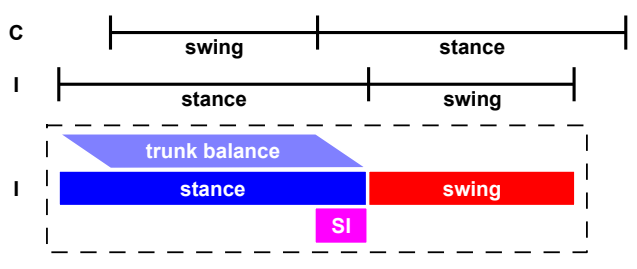

(b)

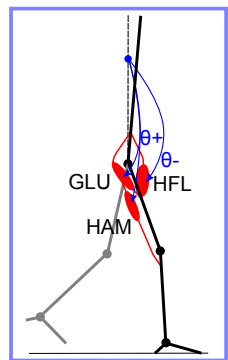

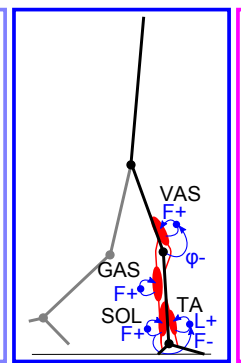
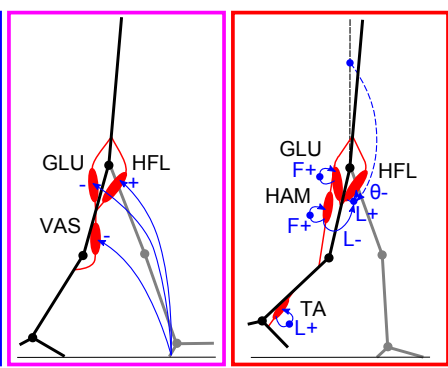

Fig. 1. The functional control groups of the previous neuromuscular model for human walking [19]. The control architecture is grouped into the control of trunk balance, stance, swing initiation (SI), and swing. The sequencing of the control groups and schematics of the active reflex pathways are shown in (a) and (b), respectively (color matched). In (a), I and C refer to the ipsilateral and contralateral leg.

The sensory feedback pathways of the stance control have been synthesized by translating a bipedal spring-mass model [25] into an articulated one [26], and encoding intrinsic stability of compliant leg behavior into muscle reflexes control. However, the swing controller of this model does not explicitly include the functionality of robust swing leg placements.

\section{B. Robust Swing Leg Torque Control}

The swing control identified in [24] is based on a double pendulum analogy of human swing legs and achieves robust swing leg placement (Fig. 2-a). It does not enforce predefined joint trajectories, but rather achieves specific functional goals. The control gets a target leg angle $\alpha_{t g t}$ and a leg clearance length $l_{c l r}$ as input commands, and is separated between the hip and knee as much as possible. The hip controller propels the leg towards target leg angle $\alpha_{t g t}$, while the knee controller follows a sequence of (i) actively flexing the knee up to the target leg clearance length $l_{c l r}$, (ii) holding the knee as the leg approaches the target angle $\alpha_{t g t}$, and (iii) stopping and extending the leg to initiate ground contact at the target angle.

In addition to $\alpha_{t g t}$ and $l_{c l r}$, the swing torque control has ten internal control parameters. Once identified these internal parameters are not changed, and the control achieves robust placement of the leg for a large range of target angles and from extreme initial angular velocities with average and maximum placement errors of $1.4^{\circ}$ and $5.2^{\circ}$, respectively (Fig. 2-b).

\section{INTEGRATION OF THE ROBUST SWING CONTROL}

To test if the proposed swing control allows to generate more robust and adaptive human locomotion behaviors, we integrate it in the neuromuscular human walking model. This extended model is not purely actuated by muscles as the swing control is implemented as an ideal torque control. The hip and knee joints are driven by the neuromuscular controller during most of the stance phase, and by the swing torque controller during the swing phase. The swing controller begins at the onset of the late double support phase, so both muscles and torque actuators are active during this phase (Fig. 3-c). The ankle by contrast is actuated by muscles throughout both phases. (The swing control did not consider the foot segment.)

In addition to the swing control integration, we modify the neuromuscular controller in three ways. First, we modify the explicit trunk control and add to the previous PDstyle control of the stance hip a feedforward control, which counters the influence of the swing leg's hip torque on trunk balance (Fig. 3-a). Second, in the previous model positive force feedbacks $F+$ of the knee and ankle extensors generates compliant leg behavior and transfers the ground reaction force to the hip. This force is large at heel strike and produces a large moment around the trunk. To reduce this moment, we add positive force feedback control to the hip extensors GLU and HAM (Fig. 3-b), better aligning the ground reaction force vector with the center of mass of the trunk. Specifically, the positive force feedback pathways are modeled as

$$
S_{m}=S_{0, m}+G_{m} F_{m}(\Delta t)
$$

for muscle $m$, where $S_{m}$ is a stimulation signal, $S_{0, m}$ is a prestimulation, $G_{m}$ is a positive force feedback gain, and $F_{m}(\Delta t)$ is the sensed muscle force delayed by $\Delta t$. ( $\Delta t$ depends on the proximity of the muscle to the spinal cord. We use $5 m s$ for both GLU and HAM.)

Third, we remove the explicit swing initiation that was required in the previous neuromuscular control (SI in Fig. 1a). Instead, the stance and swing controllers are simultaneously active during the late double support phase. The stance (a)

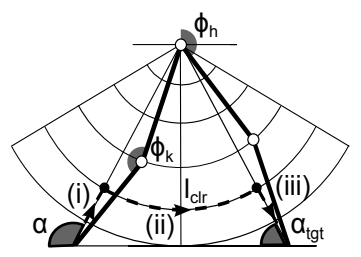

(b)

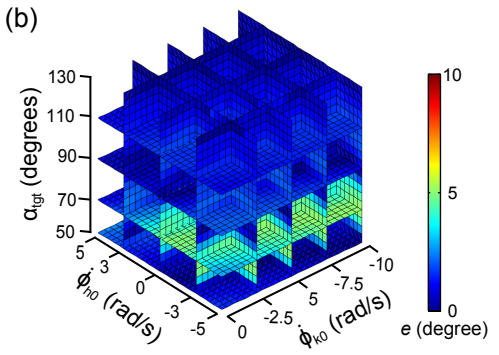

Fig. 2. Robust swing leg controller. (a) The swing controller reaches $\alpha_{t g t}$ while ensuring ground clearance $l_{c l r}$, and uses hip $\left(\phi_{h}\right)$ and knee $\left(\phi_{k}\right)$ angular data as sensory inputs. The knee controller follows a three-part control sequence (details in text). (b) The placement error $e=\left|\alpha_{t g t}-\alpha_{t d}\right|$ is shown for largely different initial angular velocities $\left(\alpha_{t d}\right.$ is leg angle at touch down). 
(a)

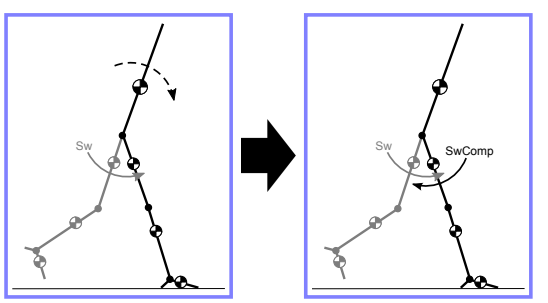

(b)

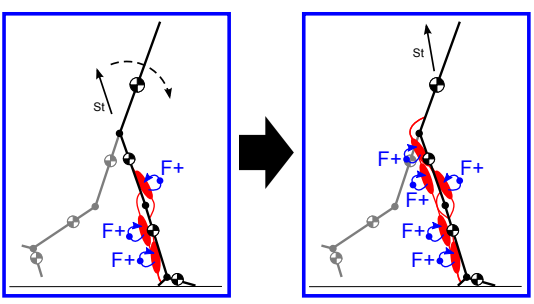

(c)

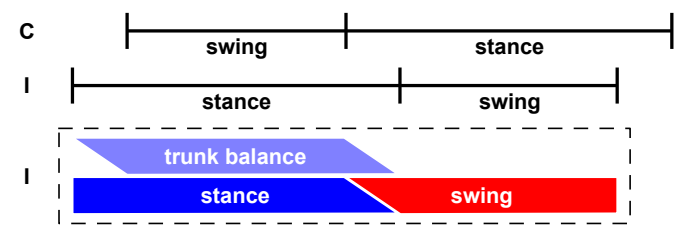

Fig. 3. Modifications made in the neurocontroller. Feedforward controllers are added to (a) the trunk balance control to compensate moments induced by the swing leg and (b) the stance control to compensate ground reaction forces transferred by the stance leg. In addition, the explicit swing initiation control required in the previous neuromuscular control is now removed (c).

control activity reduces in proportion to the load that the other (front) leg is bearing while the activity of the swing control increases by the same amount (Fig. 3-c).

\section{LOCOMOTION BEHAVIORS}

We explore the swing controller's potential for generating different locomotion behaviors including steady walking on flat ground, walking across very rough terrain, and climbing up stairs. For all three behaviors, a single set of internal swing control parameters is used based on the hand-tuned constant values identified in [24] (section II-B). In contrast, different sets of the swing control parameters $\alpha_{t g t}$ and $l_{c l r}$ as well as the stance control parameters are identified for the three individual behaviors using optimization with the covariance matrix adaptation evolution strategy (CMA-ES, [27]). The optimization uses three different cost functions for the three behaviors, samples 64 sets of the parameters based on a covariance matrix of one generation, runs individual simulations to calculate corresponding values of the cost function, and uses the best 32 sets to update the covariance matrix for the next generation. The procedure repeats for a total of 400 generations.

The cost function we use for steady walking is

$$
J_{\text {steady }}=c_{1}\left|\dot{x}_{\text {avg }}-\dot{x}_{t g t}\right|+c_{2} C_{E},
$$

where $\dot{x}_{a v g}$ and $\dot{x}_{t g t}$ are average and target walking speeds, $C_{E}$ is the energetic cost, and the coefficients $c_{1}$ and $c_{2}$ are empirically determined constants (10 and 1 , respectively). $\dot{x}_{a v g}$ and $C_{E}$ are computed during multiple consecutive steps of steady walking. $C_{E}$ is computed from $C_{E}=E_{M} /\left(m x_{d}\right)$, where $E_{M}$ is the total metabolic energy consumed by all muscles (using the energy model in [28]), $m$ is the body mass, $x_{d}$ is the walking distance traveled. For crossing very rough terrain and climbing stairs, the cost functions are defined as

$$
J_{\text {rough }}=-x_{\text {end }}
$$

and

$$
J_{\text {stair }}= \begin{cases}-c_{L}, & \text { if steady } \\ -x_{\text {end }}, & \text { o.w. }\end{cases}
$$

where $x_{\text {end }}$ is the distance travelled by the human model in the forward direction and $c_{L}=100$ is a large constant rewarding steady stair climbing.

Without changing the internal swing control parameters, the model achieves all three locomotion behaviors (Fig. 4). The model generates steady walking at normal human walking speeds $\left(1.4 m s^{-1}\right.$, Fig. 4 -a), robustly travels over terrain with randomly generated large and frequent changes in ground height (changes every $1 \mathrm{~m}$, observed maximum height changes of $+12 \mathrm{~cm}$ and $-9 \mathrm{~cm}$, Fig. 4-b), and steadily climbs stairs (arbitrarily chosen to be $50 \mathrm{~cm}$ apart and $15 \mathrm{~cm}$ high, Fig. 4-c).

\section{FUTURE DIRECTION}

The initial results show the potential of the identified swing control in generating robust and adaptive locomotion behaviors. Our long-term goal is to generalize this work and to identify a neuromuscular control architecture that combines a large range of robust and adaptive locomotion behaviors critical to humanoid and rehabilitation robotics. Toward this goal, our next step is to translate the torque controller of the swing leg into a neuromuscular one. For this, we plan to adapt the muscle-reflex control derived in [29] for the idealized double-pendulum swing leg system.

\section{REFERENCES}

[1] F. Horak, "Assumptions underlying motor control for neurologic rehabilitation," in Contemporary Management of Motor Control Problems: Proceedings of the II STEP Conference. Alexandria, Va: Foundation for Physical Therapy, 1991, pp. 11-27.

[2] H. Krebs, N. Hogan, M. Aisen, and B. Volpe, "Robot-aided neurorehabilitation," Rehabilitation Engineering, IEEE Transactions on, vol. 6, no. 1, pp. 75-87, 1998.

[3] E. Arnold, S. Ward, R. Lieber, and S. Delp, "A model of the lower limb for analysis of human movement," Annals of biomedical engineering, vol. 38, no. 2, pp. 269-279, 2010.

[4] J. Allen and R. Neptune, "Three-dimensional modular control of human walking," Journal of Biomechanics, 2012.

[5] S. Song and H. Geyer, "The energetic cost of adaptive feet in walking," in Robotics and Biomimetics (ROBIO), 2011 IEEE International Conference on. IEEE, 2011, pp. 1597-1602.

[6] J. Nakanishi, J. Morimoto, G. Endo, G. Cheng, S. Schaal, and M. Kawato, "Learning from demonstration and adaptation of biped locomotion," Robotics and Autonomous Systems, vol. 47, no. 2, pp. 79-91, 2004.

[7] P. Manoonpong, T. Geng, T. Kulvicius, B. Porr, and F. Wörgötter, "Adaptive, fast walking in a biped robot under neuronal control and learning," PLoS Computational Biology, vol. 3, no. 7, p. e134, 2007.

[8] T. Klein and M. Lewis, "A physical model of sensorimotor interactions during locomotion," J. Neural Eng., vol. 9, no. 4, 2012. 
(a)

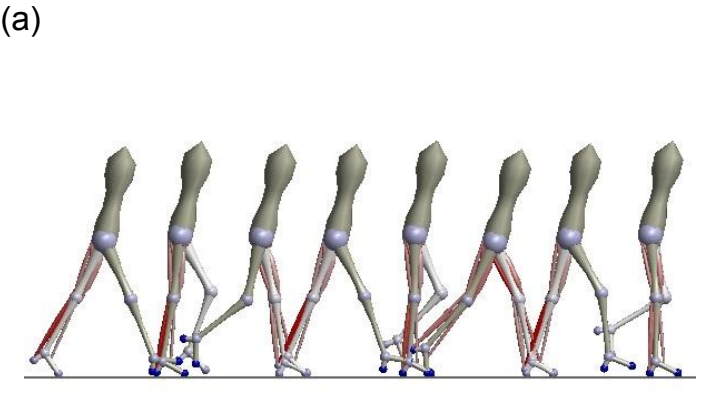

(c)

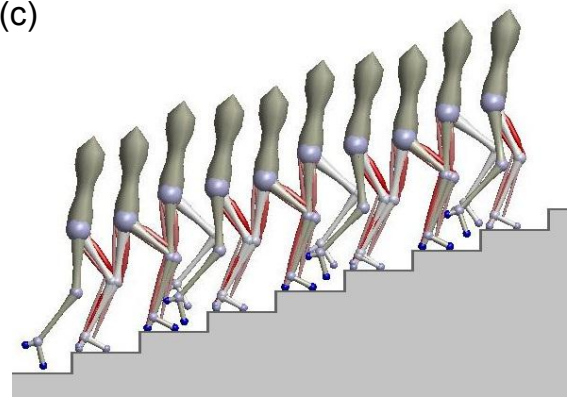

(b)

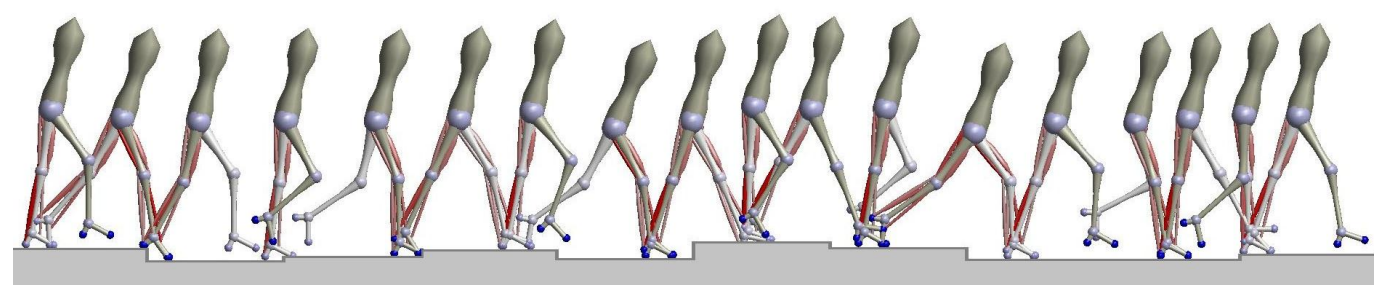

Fig. 4. Locomotion behaviors. (a), (b) and (c) show steady walking, walking across highly rough terrain, and climbing up stairs, respectively. The snapshots show poses at every $400 \mathrm{~ms}$.

[9] M. Eilenberg, H. Geyer, and H. Herr, "Control of a powered anklefoot prosthesis based on a neuromuscular model," Neural Systems and Rehabilitation Engineering, IEEE Transactions on, vol. 18, no. 2, pp. 164-173, 2010.

[10] S. Grillner, "Locomotion in vertebrates: central mechanisms and relex interaction," Physiol. Rev., vol. 55, pp. 274-304, 1975.

[11] K. Pearson and J. Ramirez, "Locomotion in vertebrates: central mechanisms and relex interaction, mit press," Sensory modulation of pattern-generating circuits, pp. 225-235, 1975.

[12] G. Taga, Y. Yamaguchi, and H. Shimizu, "Self-organized control of bipedal locomotion by neural oscillators in unpredictable environment." Biol Cybern, vol. 65, no. 3, pp. 147-159, 1991.

[13] G. Taga, "A model of the neuro-musculo-skeletal system for anticipatory adjustment of human locomotion during obstacle avoidance." Biol Cybern, vol. 78, pp. 9-17, 1998 Jan.

[14] N. Ogihara and N. Yamazaki, "Generation of human bipedal locomotion by a bio-mimetic neuro-musculo-skeletal model." Biol Cybern, vol. 84, no. 1, pp. 1-11, 2001.

[15] K. Hase, K. Miyashita, S. Ok, and Y. Arakawa, "Human gait simulationwith a neuromusculoskeletal model and evolutionarycomputation," J. Visual. Comput. Animat., vol. 14, pp. 73-92, 2003.

[16] Y. Kim, Y. Tagawa, G. Obinata, and K. Hase, "Robust control of cpg-based $3 \mathrm{~d}$ neuromusculoskeletal walking model," Biological cybernetics, pp. 1-14, 2011.

[17] M. Günther and H. Ruder, "Synthesis of two-dimensional human walking: a test of the $\lambda$-model," Biol. Cybern., vol. 89, pp. 89-106, 2003.

[18] A. Feldman et al., "Once more on the equilibrium-point hypothesis (lambda model) for motor control." Journal of motor behavior, vol. 18, no. 1 , p. 17, 1986.

[19] H. Geyer and H. Herr, "A muscle-reflex model that encodes principles of legged mechanics produces human walking dynamics and muscle activities," IEEE Trans Neural Syst Rehabil Eng, vol. 18, no. 3, 2010.

[20] S. Song and H. Geyer, "Regulating speed and generating large speed transitions in a neuromuscular human walking model," in Robotics and Automation (ICRA), 2012 IEEE International Conference on. IEEE, 2012, pp. 511-516.

[21] M. Raibert and E. Tello, "Legged robots that balance," IEEE Expert, vol. 1 , no. 4, pp. 89-89, 1986.

[22] S. Kajita and K. Tani, "Study of dynamic biped locomotion on rugged terrain-derivation and application of the linear inverted pendulum mode," in IEEE Int. Conf. on Robotics and Automation, 1991.

[23] A. Seyfarth, H. Geyer, and H. Herr, "Swing-leg retraction: a simple control model for stable running," The Journal of Experimental Biology, vol. 206, pp. 2547-2555, 2003.
[24] R. Desai and H. Geyer, "Robust swing leg placement under large disturbances," in IEEE Int. Conf. Robotics and Biomimetics, 2012.

[25] H. Geyer, A. Seyfarth, and R. Blickhan, "Compliant leg behaviour explains the basic dynamics of walking and running," Proc. R. Soc. Lond. B, vol. 273, pp. 2861-2867, 2006.

[26] — - "Positive force feedback in bouncing gaits?" Proceedings of the Royal Society of London. Series B: Biological Sciences, vol. 270, no. 1529, pp. 2173-2183, 2003.

[27] N. Hansen, "The cma evolution strategy: A comparing review," Towards a New Evolutionary Computation. Advances on Estimation of Distribution Algorithms. Springer, p. 75.102, 2006.

[28] B. R. Umberger, K. G. Gerritsen, and P. E. Martin, "A model of human muscle energy expenditure," Comp. Meth. Biomech. Biomedic. Eng., vol. 6, no. 2, pp. 99-111, 2003.

[29] R. Desai and H. Geyer, "Muscle-reflex control of robust swing leg placement," in Robotics and Automation (ICRA), 2013 IEEE International Conference on. IEEE, accepted. 